\title{
A new method to eliminate dead zone of phase-comparison instruments adjacent to $0^{\circ}$ and $360^{\circ}$
}

\author{
Yuzhen $\mathrm{Jin}^{1, \mathrm{a}}$, Zongqiang Xuan ${ }^{2, \mathrm{~b}}$ and Fuming Wang ${ }^{3, \mathrm{c}}$ \\ ${ }^{1}$ Xidian University, Xi'an, 710071, China \\ ${ }^{2}$ Xidian University, Xi'an, 710071, China \\ ${ }^{3}$ Xidian University, Xi'an, 710071, China \\ ajinyuzhen91@126.com, bxzq6002@126.com, ${ }^{\circ} 517804551 @ q q . c o m$
}

Keywords: Phase-comparison Curve; High Linearity; No Dead-zone.

\begin{abstract}
With the development of Modern High-New Techniques, the high-precision phase comparison instruments play a great role in navigational positioning, network communication, field of national defense etc. So it is important to resolve the phenomenon of "dead zone" during phase comparison. In this paper, a method of high-precision phase comparison between periodic signals is proposed based on phase coincidence theory and border effect theory. By this way, the \pm 1 count error can almost be eliminated. Above all, a phase-comparison curve with high performance such as full period, no dead-zone and high linearity, is acquired. The experimental result proves that this method is reliable and practical, meanwhile the phase-comparison curve is of significance in other measurement fields.
\end{abstract}

\section{Introduction}

The high-precision phase comparison apparatus is widely used in communication system, time service, comparison of frequency standard, field of broadband infrastructure etc., especially in the study of phase frequency characteristics of the network ${ }^{[1]}$. Furthermore, since ultra-high precision frequency sources come out gradually, the theory of measuring and processing time-frequency signals also needs to be kept reforming and innovating, then there is a need to strengthen the research on ultra-high precision phase comparison devices ${ }^{[2]}$. In the process of the rapid development of modern science and technology, phase difference is usually measured by observing oscilloscope, comparing phase, measuring voltage and measuring time interval. These common methods can be utilized in some fields without the requirement of high precision. But, because of the limitation of these methods themselves, leading to the impossibility of improving the measuring precision ${ }^{[3]}$. So it is extremely urgent to do research on new method of phase comparison to meet the requirement of high precision in every field. By a study of precise time and frequency measurement technology, it is useful to increase the precision in phase comparison measurement by combing the theory of relationship between phases and the theory of border effect, which contributes to advancing the development of measurement fields. On the basis of these two theories, a system of phase comparison is designed for acquiring a phase-comparison curve with high linearity and full period. 


\section{Basic theories}

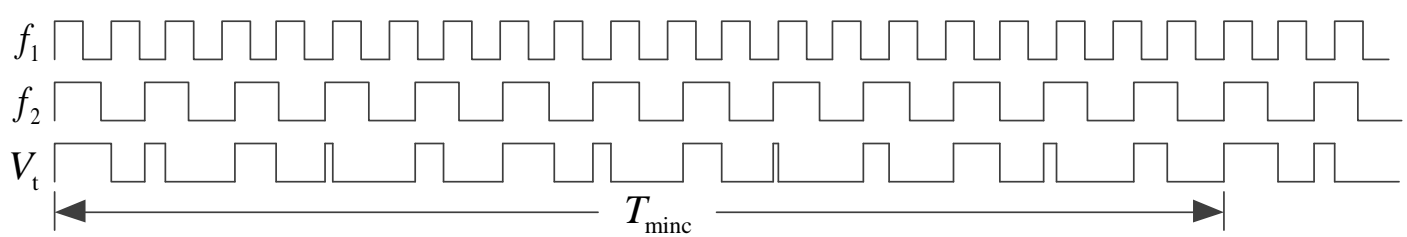

Fig. 1 Phase relationship between periodic signals

The greatest common factor frequency $f_{\operatorname{maxc}}$ and the least common multiple period $T_{\operatorname{minc}}$ are adopted to reflect the cyclic variation of phase difference between two periodic frequencies ${ }^{[4]}$. They are defined as follows:

$$
f_{1}=A \cdot f_{0} f_{2}=B \cdot f_{0}
$$

$f_{1}$ and $f_{2}$ are two arbitrary signals. $A$ and $B$ are co-prime, then $f_{0}$ is the greatest common factor frequency of $f_{1}$ and $f_{2}$ and defined as $f_{\operatorname{maxc}}$. $T_{\text {minc }}$ is defined as the period of $f_{\operatorname{maxc}}$. According to Eq. 1 , it can be known that $T_{\text {minc }}$ is an integral multiple of signal $f_{1}$ and $f_{2}$. The $f_{\operatorname{maxc}}$ is a periodic characteristic value which represents the relationship between two compared signals and is the basis to analyze the relationship between two compared signals.

$$
\Delta T=\frac{f_{\text {maxc }}}{f_{1} f_{2}} .
$$

The Eq. 2 given above defines that $\Delta T$ is the quantitative step value of phase difference. $\Delta T$ represents the least change in phase difference and it is a constant if $f_{1}$ and $f_{2}$ are given. The precision of phase comparison measurement will be high by increasing values of compared signals while the value of the greatest common factor frequency is little.

The phase difference of two periodic signals changes with time periodically and the period of variation is the the least common multiple period $T_{\text {minc }}$. "phase coincidence point" is defined as the state when phases of two periodic signals are completely or almost coincident. In one $T_{\operatorname{minc}}$, the phase difference of two signals range in $0 \sim \Delta T^{[5]}$. Using the detection technology of phase coincidence point can acquire coincidence points when phase difference is less than or equal to $\Delta T$.

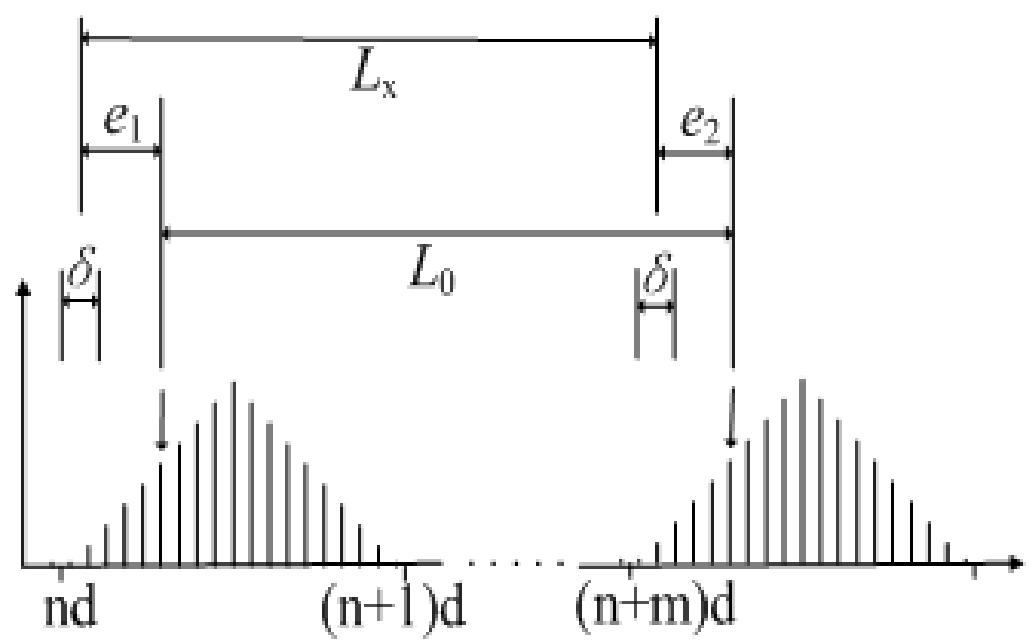

Fig. 2 Schematic diagram of border effect 
For the limited measurement resolution of devices, it is hard to capture phase coincidence points accurately for obtaining high precision. However, the detective resolution is more stable at the border. So, when the coincident states come out, capturing borders in two adjacent measurements and the error will cancel each other out, then high precision of measurement is obtained. The detailed description of Fig. 2 is in reference [6].

\section{Measurement principle}

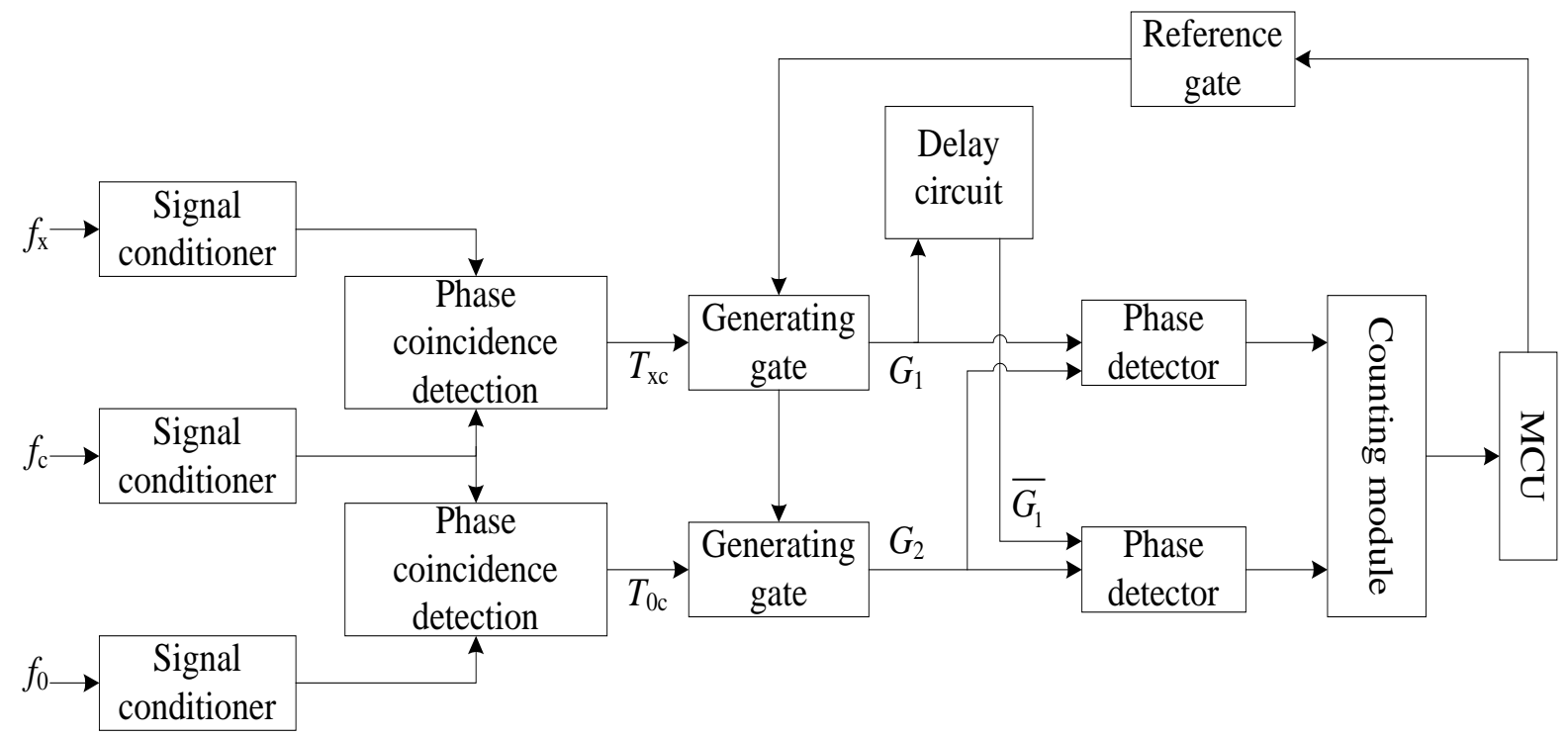

Fig. 3 The block diagram of the phase-comparison system

In this measurement system, $f_{\mathrm{c}}$ is a high-stability common frequency source, and it is almost a multiple of $f_{0}$ which is a standard frequency signal. The $f_{\mathrm{x}}$ is a measured frequency signal. Comparing $f_{\mathrm{c}}$ with $f_{\mathrm{x}}$ and $f_{0}$ separately, then it is good to avoid comparing $f_{\mathrm{x}}$ with $f_{0}$ directly. Only when $f_{\mathrm{c}}$ is suitable will it realize high-precision measurement of phase difference between signals with the same frequency. Meanwhile, for $\Delta T$ of $f_{\mathrm{x}}$ and $f_{0}$ is little, so the requirement of the circuit is low and the measurement precision is still high.

The block diagram of the phase-comparison system is shown in Fig. 3. Phase coincidence points of $T_{\mathrm{xc}}$ and $T_{0 \mathrm{c}}$ are controlled by reference gate to generate true gates $G_{1}$ and $G_{2}$. One phase detector is opened by $G_{1}$ and closed by $G_{2}$ and the other one is opened by $\overline{G_{1}}$ and closed by $G_{2}$, that $G_{1}$ is delayed $180^{\circ}$ to get $\overline{G_{1}}$. When counters are filled with pulses $f_{\mathrm{c}}$ or $f_{\mathrm{x}}$, the rising edge of pulses will be counted. Because essentially it is $f_{\mathrm{c}}$ that triggers phase detectors on and off, and $f_{\mathrm{x}}$ just triggers phase detectors on. So count values of $f_{\mathrm{c}}$ are integers, but that of $f_{\mathrm{x}}$ are not integers, as a result phase difference can be calculated.

Suppose $N_{\mathrm{c} 1}$ and $N_{\mathrm{x} 1}$ are recorded by one counter, then $N_{\mathrm{c} 2}$ and $N_{\mathrm{x} 2}$ are recorded by the other one. $T_{\mathrm{c}}$ and $T_{\mathrm{x}}$ are periods of $f_{\mathrm{c}}$ and $f_{\mathrm{x}} . \Delta t_{1}$ and $\Delta t_{2}$ are defined as follows:

$$
\begin{aligned}
& \Delta t_{1}=N_{\mathrm{c} 1} T_{\mathrm{c}}-N_{\mathrm{x} 1} T_{\mathrm{x}} . \\
& \Delta t_{2}=N_{\mathrm{c} 2} T_{\mathrm{c}}-N_{\mathrm{x} 2} T_{\mathrm{x}} .
\end{aligned}
$$

The phase difference of $f_{\mathrm{x}}$ and $f_{0}$ are $v t_{1}$ and $v t_{2}$, and they are acquired as follows:

$$
v t_{1}=\Delta t_{1} / T_{\mathrm{x}} \times 360^{\circ} \text {. }
$$




$$
v t_{2}=\Delta t_{2} / T_{\mathrm{x}} \times 360^{\circ}
$$

In traditional methods of phase detection, when devices are in the state of on and off, it is impossible to output a perfect square wave with an input of high frequency. If the phase difference of two measured frequencies is away from $0^{\circ}$ and $360^{\circ}$, phase detectors output a curve with good linearity. But when the phase difference is near $0^{\circ}$ or $360^{\circ}$, phase detecting circuit is hard to respond, leading to lose some triggering information. This phenomenon contributes to the result of non-linearity and dead zone, which has an influence on measuring accuracy. Under this case, counting error will also come out when phase-difference signals are filled with pulses. It is difficult to implement high linearity and no dead-zone during phase comparison. The method of dual phase detection is adopted in the system to combine two phase-comparison curves, then what is got is a high-linearity curve ranging from $0^{\circ} \sim 360^{\circ}$.

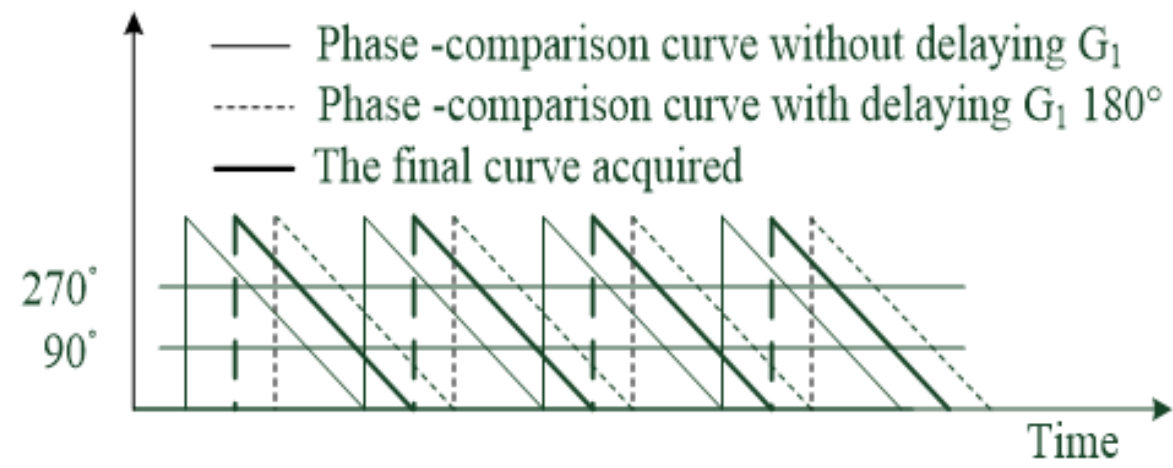

Fig. 4 The schematic diagram of acquiring a high-performance phase-comparison curve

\section{Experiment result}

In the experiment, comparing two different source signals, the common frequency source is generated by signal generator HP8662A. The measured frequency signal is output by cesium atomic clock OSA5585B and the standard frequency signal is output by rubidium atomic clock. The experimental result is shown in Fig. 5.

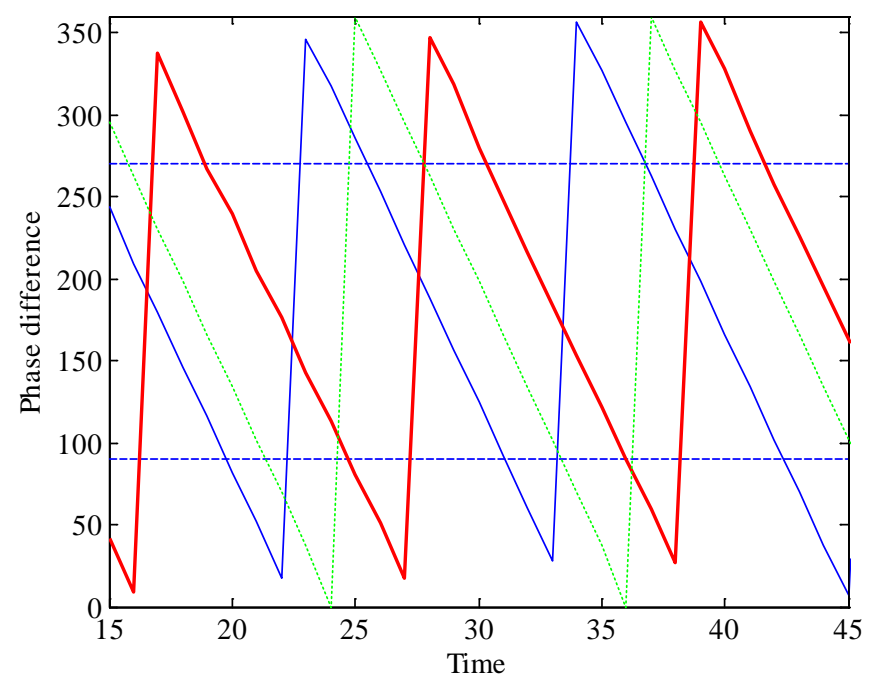

Fig. 5 The result of experiment

It can be seen that the result of this experiment is in perfect accordance with the principle. Choosing the region $\left(90^{\circ} \sim 270^{\circ}\right)$ of two curves and these two parts create a new phase-comparison curve. In this new curve, dead zone nearly disappear. So the method proposed in this paper solves 
problems of non-linearity and dead zone well, and it saves hardware resources effectively.

\section{Conclusion}

Traditional methods of phase comparison switch phase difference signals to voltage signals for acquiring phase-comparison curves to calculate phase difference . While in this paper, firstly, using detection technology of phase coincidence theory to detect phase coincidence points, secondly phase coincidence pulses work as inputs of phase detectors. Then a signal that equals to phase difference signal is obtained with large pulse width. So it is convenient to fill the phase difference signal with pulses. Because of a $180^{\circ}$ delay between two phase-comparison curves, it can be done to combine the $90^{\circ} \sim 270^{\circ}$ region of these two curves for obtaining a phase -comparison curve with high linearity and no dead-zone. This method has advantages of high precision, simple devices, easy operation, etc. It do play an important role in technology of the precise comparison of frequency standard.

\section{References}

[1] G. Li, Z.M. Wang and J. Lv: J. Mil. Comm. Tech. Vol. 30 (2009), p. 94

[2] B.Y. Huang, in: Handbook of Measurement Technology, edited by Editorial Board, volume 11 of Time and Frequency, chapter, 1, Chinese Metrology Publisher (1996).

[3] S.F. Dong, W. Zhou, B.Q. Du and C.Z. Jiao, Ultra-high Resolution Phase Difference Measurement Method, IEEE Conference on Frequency Control Symposium, (2012).

[4] W. Zhou, The Greatest Common Factor Frequency and Its Application in the Accurate Measurement of Periodic Signals, IEEE Conference on Frequency Control Symposium, (1992).

[5] Y.X. Ye, Z.Q. Xuan, J.S. Gu and Y. Xuan: Chin. Phys. B Vol. 23 (2014), 120601.

[6] W. Zhou, Z.Q. Li, L.N. Bai, Z.Q. Xuan, F.X. Chen, J.G. Yu, J.N. Gao, M. Miao, S.F. Dong, H.M. Song, Z. Wei and Y.X. Ye: Chin. Phys. Lett. Vol. 31 (2014), 100602. 